\title{
La pandemia COVID-19 y el mundo de la ecocardiografía
}

I 31 de diciembre de 2019 se comunicaron varios brotes de neumonía de causa desconocida que compartían el antecedente epidemiológico de cercanía a un mercado de animales vivos en la ciudad china de Wuhan. El 9 de enero de 2020 se identificó como causante a un nuevo coronavirus, que recibiría el nombre de SARS-CoV-2 $2^{(1,2)}$. Los coronavirus son una familia de virus que tienen una proteína de superficie que causa prominencias o espinas que recuerdan a una corona en la imagen del microscopio electrónico. La enfermedad quedó oficialmente bautizada como COVID-19. El día 11 de marzo, la Organización Mundial de la Salud (OMS) declaró la pandemia mundial. Desde el inicio de la epidemia a la fecha de este resumen se han contabilizado más de 500.000 fallecimientos en el mundo y detectado cerca de 10.000 .000 de casos.

Sin duda, la pandemia ha cambiado y va a modificar muchos aspectos de nuestra vida diaria. El mundo de la Cardiología y, especíicamente, el de la imagen cardíaca igualmente se ha visto muy afectado por la misma. Nos parece obligado dejar constancia también en nuestra revista RETIC de la importante tormenta que nos afecta y dejar por escrito lo que fue, lo que será y lo que es, la influencia de COVID-19 en nuestra vida de expertos en imagen.

\section{COVID-19 y corazón}

El corazón puede verse afectado ya que la lesión cardíaca aguda, el shock y la arritmia están presentes en el 7,2\%, 8,7\% y 16,7\% de los pacientes, respectivamente, con una mayor prevalencia entre los pacientes que requieren ingreso en Cuidados Intensivos ${ }^{(3)}$ :

- Enfermedad coronaria y COVID-19. Los pacientes con enfermedad coronaria e insuficiencia cardíaca pueden tener un riesgo particular como resultado de la ruptura de la placa coronaria secundaria a la inflamación sistémica inducida por el virus. Los efectos procoagulantes de la inflamación sistémica pueden aumentar igualmente el riesgo de patología coronaria. La probabilidad de trombosis del stent es también una posibilidad que hay que considerar.

- Miocarditis y COVID-19. El virus SARS-CoV-2 puede afectar al miocardio y producir miocarditis. Se han publicado casos esporádicos de autopsia que han mostrado alteración del miocardio por células inflamatorias mononucleares. Aunque es una posibilidad poco frecuente, puede estar ligada tanto a daño directo del miocardio por el virus, como a un daño secundario a la cascada inflamatoria(4,5). El pronóstico es bueno y si el paciente sobrevive, se puede recuperar la función miocárdica. Aunque las series publicadas hasta el momento son pequeñas y en algún caso está descrita una miocarditis fulminante.

- Arritmias y COVID-19. Es uno de los motivos más frecuentes de interconsulta del área de Intensivos a Cardiología en nuestro medio. En 138 pacientes hospitalizados con COVID-19, las arritmias representaron la complicación principal (19,6\%) y muy específicamente en aquellos ingresados en la Unidad de Cuidados Intensivos donde la prevalencia aumentó a 44,4\%(6); arritmias ventriculares malignas, como la taquicardia/ fibrilación ventricular (TV/FV) en el 5,9\% de los casos. Actualmente, se cree que el daño miocárdico podría representar un factor principal del aumento del riesgo arrítmico en estos pacientes, como se demuestra por el aumento de los niveles de troponina. Así en los pacientes con estas enzimas elevadas es más frecuente la presencia de taquicardias malignas ${ }^{(7)}$. Los mecanismos de lesión miocárdica aún están bajo investigación, probablemente tengan relación con la infección viral directa, así como con la lesión celular relacionada con la tormenta de citocinas. Sin embargo, más de la mitad de los pacientes con arritmias malignas tienen las troponinas normales, por lo que se ha alertado sobre la posibilidad de que las arritmias puedan ser un efecto secundario de los fármacos empleados como la cloroquina y la hidroxicloroquina, otros agentes antimaláricos, el uso de inhibidores de la proteasa que inhiben la replicación del ARN del virus, o macrólidos como la azitromicina que, como es conocido, producen un alargamiento del QT ${ }^{(8)}$.

- Daño miocárdico y COVID-19. En series de pacientes hospitalizados por COVID-19 se muestra que una quinta parte tenía daño cardíaco demostrado por elevación de la troponina ultrasensible ${ }^{(9,10)}$. Estos pacientes eran más mayores, tenían más comorbilidades y tenían cifras más altas de leucocitos, proteína C reactiva y procalcitonina. Los pacientes con elevación de troponina tenían una proporción mucho mayor de distrés respiratorio (58,5\% frente a 14,7\%) y una mortalidad más elevada (51,2\% frente a 4,5\%). Por tanto, los pacientes con troponina elevada marcan un grupo de más riesgo de complicaciones. 


\section{COVID-19 y datos ecocardiográficos}

La realización de estudios ecocardiográficos en una gran serie multicéntrica reciente muestra que la ecocardiografía es anormal en prácticamente la mitad de los pacientes, siendo las alteraciones más frecuentes la presencia de anomalías en el ventrículo izquierdo (39\%), datos sugerentes de infarto agudo de miocardio en el 3\% de los casos, miocarditis en el 3\% y un 2,5\% la enfermedad de tako-tsubo. El compromiso importante por taponamiento o disfunción ventricular severa ocurre en el $15 \%$ de los $\operatorname{casos}^{(11)}$.

Obviamente, los hallazgos ecocardiográficos pueden venir matizados por la presencia de enfermedad cardíaca previa, más aún teniendo en cuenta que la severidad de la afectación del virus está en directa relación con la edad del paciente; así, entre las grandes series chinas, un 4,2\% de los pacientes tenía antecedentes de enfermedad cardiovascular de base ${ }^{(3)}$. En estos pacientes se agrupó un 22,7\% de todos los casos fatales. La mortalidad en pacientes con hipertensión fue del 6\%, en pacientes con diabetes del 7,3\% y en pacientes con enfermedad respiratoria crónica del 6,3\%. En series más pequeñas se ha comprobado que entre pacientes ingresados con COVID-19 el porcentaje de pacientes con antecedentes cardiovasculares llega hasta el 40\%, incluyendo enfermedad cardiovascular (15\%), hipertensión (15\%) y diabetes (20\%).

\section{Los laboratorios de ecocardiografía durante la pandemia}

Los escenarios clínicos a los que se ha hecho y se sigue haciendo frente han sido muy diversos y, en muchas situaciones, dramáticos.

Por un lado, están los estudios en pacientes con COVID-19 que han "Ilenado" los laboratorios en la época del pico de la pandemia. La realización del estudio es compleja desde el punto de vista de la protección, dado que resulta imposible mantener una distancia mínima de seguridad con el paciente, y el examen ecocadiográfico se convierte en uno de los de mayor riesgo para los profesionales de la salud. En los pacientes con COVID-19 que necesitan un estudio en el laboratorio de ecocardiografía, la mejor opción ha sido habilitar una sala y un circuito específico (circuito COVID). De no ser así, en estos pacientes es recomendable llevar la ecocardiografía a donde se encuentren, bien utilizando equipos portátiles o, si es posible, utilizando equipos que pueden estar disponibles en distintas áreas del hospital (urgencias, intensivos, quirófano), con el objetivo de minimizar el riesgo de contagio entre pacientes. Cuando se acercan los equipos de ecocardiografía a los pacientes enfermos es importante que el médico que realiza la exploración tenga la formación necesaria en ecocardiografía y que los equipos tengan la capacidad de almacenar las imágenes obtenidas para que puedan reevaluarse en el laboratorio de ecocardiografía si fuese necesario.

Por otro lado, en la fase de vuelta a la normalidad de los distintos laboratorios de ecocardiografía, se encuentran pacientes en los que no se sabe si la infección está presente. Probablemente éstos sean los estudios donde haya que pensar mejor cómo actuar y protegerse. No sólo por la posibilidad de que el virus se transmita al personal sanitario, sino porque los laboratorios pueden convertirse en un foco de transmisión del virus.

La SEIC y la SISIAC, al igual que otras sociedades científicas ${ }^{(12-14)}$, han emitido documentos de consenso insistiendo en la importancia de realizar un estudio con las máximas garantías. Por un lado, sigue siendo muy importante establecer la adecuada indicación del mismo y, por otro, es vital realizar los estudios en un ambiente de trabajo seguro tanto para proteger al personal sanitario como para evitar la transmisión del virus entre pacientes, y es que a pesar de que se sigue insistiendo en la protección del personal sanitario, la realidad es que el mismo está muy expuesto. Como ejemplo, España contabilizaba alrededor del mes de mayo un total de 45.000 profesionales sanitarios contagiados, una cifra que representa casi el $20 \%$ del total de positivos en el país. Estas cifras obviamente son cambiantes según el devenir de la pandemia. En países donde el estudio ecocardiográfico es realizado por sonocardiografistas, éstos se comportan como una población de alto riesgo por las razones comentadas previamente.

En resumen, una inadecuada protección puede llevar y ha llevado a un mayor contagio entre los profesionales sanitarios, a que la enfermedad se siga propagando desde los laboratorios y a errores diagnósticos que pueden resultar fatales en el manejo de los pacientes. Por tanto, es obligatorio implementar medidas como lavarse adecuadamente las manos tras la realización de cada estudio y usar adecuadamente el material de protección indicado.

\section{La ecografía pulmonar explota como método diagnóstico}

Es llamativo cómo la pandemia COVID-19 ha relanzado el uso de recursos que no estaban integrados de una manera rutinaria en los laboratorios de ecocardiografía. Un ejemplo claro ha sido la utilización y puesta de largo de la ecografía pulmonar. 
Aunque muchos hemos abogado por su utilización rutinaria en la exploración del paciente cardíaco y a pesar de la extensa literatura, no sólo en el medio de medicina crítica y de urgencia, sino también en la medicina cardiovascular, no ha sido hasta esta pandemia y dada su utilidad en el triaje del enfermo COVID-19 con afectación pulmonar, cuando la ecografía pulmonar se ha introducido en su uso rutinario en muchos laboratorios. Es llamativa la explosión de recursos de formación en el área del uso de la ecografía pulmonar, lo que sin duda va a ser el pilar más importante para que ésta no abandone el escenario cardiovascular en el que tanto le ha costado entrar.

\section{La formación y comunicación científica en el mundo COVID}

La repercusión que esta pandemia está teniendo en la formación médica está siendo muy importante y muestra la capacidad de los profesionales para reciclarse y adaptarse a las adversidades. De un plumazo se han anulado cursos, reuniones, prácticas hospitalarias de másteres o entrenamientos. Esto ha sido así en todo el mundo médico y afecta igualmente en la ecocardiografía y al área de la imagen cardíaca.

Dentro de los nuevos modos de hacer, ha nacido la época de transmisión online de reuniones, nos hemos "zoomizado" en honor a uno de los máximos exponentes de las trasmisiones científicas. La formación online en cada una de sus variantes ha sufrido un salto exponencial. Igualmente, ahora se ha demostrado que las reuniones online de expertos en todas sus modalidades, que ahora son obligadas, tienen su utilidad real y Ilenan plenamente la ausencia de congresos presenciales. Obviamente se pierde el componente humano de reencuentro directo entre colegas, que sin duda es una parte importante de las reuniones, pero se gana en muchas cosas como son el contacto más sencillo, el ahorro de recursos, la facilidad de los contactos... En mi opinión, va a producirse una revolución en la formación. Los congresos y las reuniones se van a modificar en el inmediato, cercano y en el próximo futuro y sólo el tiempo dirá hasta dónde llegará esta revolución en la formación e intercambio científico y médico. Este cambio era necesario y la pandemia no ha hecho otra cosa que acelerarlo.

\section{Conclusión}

Es un tiempo difícil para todos. Hemos pasado a vivir situaciones reales que hace sólo unos meses nos habrían parecido una exageración de un escritor de novela de ficción. Creo que podemos estar orgullosos de la actuación de nuestros sanitarios, nadie mejor que nosotros para admirar su trabajo.

La luz del túnel surge ya en muchos países, no sé si las cosas volverán a ser iguales, pero debemos seguir haciendo el esfuerzo para extraer de esta situación extrema lo mejor para los enfermos, que es el fin de nuestro trabajo diario.

Gracias a todos los que han participado en este esfuerzo, que muchas veces ha sido más que titánico, su actuación es un honor para todos.

Miguel Ángel García Fernández Catedrático del Departamento de Medicina-Imagen Cardíaca. Universidad Complutense de Madrid. Presidente de la Sociedad Española de Imagen Cardíaca (SEIC)

Citar como: García-Fernández MA. La pandemia COVID-19 y el mundo de la ecocardiografía. Rev Ecocar Pract (RETIC). 2020 (Jul); 3 (2): I-IV. doi: 10.37615/retic.v3n2a1.

Cite this as: García-Fernández MA. The COVID-19 pandemic and the world of echocardiography. Rev Ecocar Pract (RETIC). 2020 (Jul); 3 (2): I-IV. doi: 10.37615/retic.v3n2a1. 


\section{Bibliografía}

1. Lu H, Stratton CW, Tang Y. Outbreak of pneumonia of unknown etiology in Wuhan, China: The mystery and the miracle. Journal of Medical Virology 2020; 92 (4): 401-402.

2. Hui DS, Azhar E, Madani TA, et al. The continuing 2019-nCoV epidemic threat of novel coronaviruses to global health - The latest 2019 novel coronavirus outbreak in Wuhan, China. Int J Infect Dis 2020; 91: 264-266.

3. Wang D, Hu B, Hu C, et al. Clinical characteristics of 138 hospitalized patients with 2019 novel coronavirus-infected pneumonia in Wuhan, China. JAMA 2020; 323 (11): 1061

4. Zeng JH, Liu Y, Yuan J, et al. First case of COVID-19 infection with fulminant myocarditis complication: Case report and insights. Preprints $2020 ; 2020030180$.

5. Hu H, Ma F, Wei X, Fang Y. Coronavirus fulminant myocarditis treated with glucocorticoid and human immunoglobulin. European Heart Journa Oxford University Press (OUP) 2020. Disponible en: https://doi. org/10.1093/eurheartj/ehaa190 PMID:32176300

6. Lazzerini PE, Boutidor M, Capechi PL. COVID-19, arrhythmic risk and in ammation: Mind the Gap! Circulation 2020 (in press). Disponible en: https:/doi org/10.1161/ CIRCULATIONAHA.120.047293

7. Haeusler IL, Chan XH, Guérin PJ, White NJ. The arrhythmogenic cardiotoxicity of the quinoline and structurally related antimalarial drugs: a systematic review. BMC Med 2018; 16 (1): 200.

8. Fossa AA, Wisialowski T, Duncan JN, et al. Azithromycin/chloroquine combination does not increase cardiac instability despite an increase in monophasic action potential duration in the anesthetized guinea pig. Am J Trop Med Hyg 2007; 77 (5): 929-938.

9. Lippi G, Lavie CJ, Sanchis-Gomar F. Cardiac troponin I in patients with coronavirus disease 2019 (COVID-19): evidence from a meta-analysis. Prog Cardiovasc Dis 2020; S0033-0620(20)30055-4.

10. Shi S, Qin M, Shen B, et al. Association of Cardiac Injury With Mortality in Hospitalized Patients With COVID-19 in Wuhan, China. JAMA Cardiol 2020.

11. Dweck M, Bularga A, Hahn RT, et al. Global evaluation of echocardiography in patients with COVID-19. European Heart Journal - Cardiovascular Imaging 2020; 0: 1-10.

12. García Fernández MA, Cabrera M, Schulmeyer P, Azcárate A. Actualización de las Recomendaciones sobre el uso de la Ecocardiografía durante la Pandemia de COVID-19. Soc. Esp. de Imagen Cardíaca 2020.

13. COVID-19 Clinical Guidance For the Cardiovascular Care Team document. ACC. Disponible en: https://www.acc.org// /media/Non-Clinical/Files-PDFs-Excel-MSWord-etc/2020/02/S20028-ACC-Clinical-Bulletin-Coronavirus.pdf

14. Documento ad uso degli operatori di ecografia cardiovascolare per covid-19. SIEC. Disponible en: https://www.siec.it/documento-ad-uso-degli-operatori-di-ecografia-cardiovascolare-per-covid-19/ 